\title{
A Neuroanatomical Basis for the Frequency of Discrete Spontaneous Activities in Schizophrenia
}

\author{
Tom F.D. Farrow*, ${ }^{*}$, Michael D. Hunter ${ }^{\mathrm{a}}$, Iain D. Wilkinson ${ }^{\mathrm{b}}$ and Sean A. Spence ${ }^{\mathrm{a}}$ \\ ${ }^{a}$ Academic Clinical Psychiatry, University of Sheffield, The Longley Centre, Northern General Hospital, Norwood \\ Grange Drive, Sheffield S5 7JT, UK; ${ }^{b}$ Academic Unit of Radiology, University of Sheffield, C-Floor, Royal Hallamshire \\ Hospital, Glossop Road, Sheffield. S10 2JF, UK
}

\begin{abstract}
Limited behavioural repertoire impacts quality of life in chronic schizophrenia. We have previously shown that the amount of movement exhibited by patients with schizophrenia is positively correlated with the volume of left anterior cingulate cortex and that this quantity of movement can be increased by modafinil. However, increased movement in itself may be of limited clinical significance. Hence, we sought to analyse the 'structure' of spontaneous movement in patients with schizophrenia and to examine whether the chunking of spontaneous activity has a neuroanatomical basis. 'Actiwatches' were used to record spontaneous motor activity over a 20 hour period in sixteen male patients with schizophrenia. Time-series data were analysed for the number of discrete spontaneous activities, which might indicate a degree of structure to ongoing activity. Subjects underwent a whole-brain structural MRI scan. The 'number of discrete movement epochs' correlated with volumes of regions within bilateral rostro-ventral putamen and temporal poles. These data suggest that in people with schizophrenia the volume of bilateral putamen may influence the complexity of their behaviours, as distinct from the overall amount of behaviour. The results are presented in the context of a large body of previous research examining the role of the basal ganglia in motor and cognitive pattern generation.
\end{abstract}

\section{INTRODUCTION}

Patients with schizophrenia often show marked avolition, an inability to initiate and persist in goal-directed activities [1]. Conversely, they may also exhibit increased repetitive, stereotypic patterns of behaviour, which are not goaldirected. We have previously demonstrated that the amount of movement exhibited by patients with schizophrenia is positively correlated with the volume of their left anterior cingulate cortex [2] and that this quantity of movement can be increased by acute administration of modafinil (a putative cognitive enhancer) [3]. However, movement in itself is not enough; e.g. stereotypic movement lacks purpose, and in patients with schizophrenia, akathisia, an inner restlessness characterised by an inability to remain still, can be provoked as a side-effect of prescribed anti-psychotic medication [4]. Akathisia certainly precipitates movement but it is classically stereotypic and unproductive. Hence, in order to study volition quantitatively, what is needed is a way of discerning whether ongoing activity in people with schizophrenia is purposeful or not.

Classically, the control of ongoing movements is modulated by the basal ganglia, a group of nuclei comprising the corpus striatum, globus pallidus, subthalamic nucleus and substantia nigra, located close to the base of the brain. While the caudate (together with the nucleus accumbens) is implicated in coding environmental cue properties, the putamen is implicated in the subsequent motor response [5].

*Address correspondence to this author at the Sheffield Cognition and Neuroimaging Laboratory (SCANLab), Academic Clinical Psychiatry, University of Sheffield, The Longley Centre, Northern General Hospital, Norwood Grange Drive, Sheffield S5 7JT, UK; Tel: +44 (0)114 226 1511; Fax: +44 (0)114 226 1522; E-mail: t.f.farrow@ @heffield.ac.uk
The basal ganglia therefore have a fundamental influence on motor and cognitive pattern generators [6]. Their role in the production of habitual or automatic responses or habits and in stimulus-response learning may be due to different parts being specialised for the acquisition and expression phases of motor learning [7]. A PET imaging study found that whilst the caudate (along with prefrontal and anterior cingulate cortex) was involved in new learning, the putamen (along with premotor and motor cortex) was activated during the performance of already learned sequences [8, 9]. Hence the striatum would appear to be central to both the acquisition and expression of complex motor sequences or sequential behaviour.

Avolition might be conceptualised as a deficit of 'instrumental conditioning'; how organisms choose to act in response to the affective structure of their environment. 'Reinforcement learning', which underpins instrumental conditioning, concerns how the rewards (positive or negative) for such actions are incorporated into future behaviour [10]. The neuroanatomical bases of instrumental conditioning and reinforcement learning have been demonstrated by neurophysiological (single-neuron recordings; [11], lesional and fMRI [10] studies to lie within the dorsal and ventral striatum, as well as involving the limbic system [12]. Yamada and colleagues [11] examined the role of tonically active neurons (presumed to be cholinergic interneurons) in the caudate and putamen in encoding for goal-directed action planning and learning. Whilst the caudate was implicated in responding to stimuli associated with motivational outcomes, the putamen was more involved in responding to initiation of movement toward goal-directed activities, especially those actions with an expected reward. There is also evidence from animal models that the degree of stereotypy / poverty of 
movement (and probably, by implication, the repetitive, inflexible patterns of attention, emotion, planning and cognition seen in many human neuropsychiatric disorders) may be linked to neurochemical imbalances between distinct striatal compartments [13].

In an fMRI study of instrumental conditioning in humans, distinct, dissociable functions for the dorsal and ventral striatum were investigated using a varying-reward task [10]. Whilst the ventral striatum was associated with a 'critic' role (learning to predict future rewards) the dorsal striatum was associated with an 'actor' role (maintaining information about these reward outcomes to modulate future choices). Lehéricy and colleagues [14] used fMRI to examine the role of motor, premotor and associative basal ganglia areas in the complexity and frequency of movement. However, in this audio-paced finger-tapping task study, "complexity" of movement refers to relative task difficulty (i.e. repetitive index-finger tapping versus a complex sequence of 10 finger movements) in contrast to how we might characterise complexity, behaviourally, as the structure of discrete, internally generated spontaneous activities. Lehéricy and colleagues [14] reported increasing signal in the posterior putamen in association with increased movement frequency, though not task difficulty, and increasing signal in anterior putamen with both increased movement frequency and complexity.

The basal ganglia as a whole are also involved in "chunking", the grouping together of representations of motor and cognitive action sequences into 'performance units', which may make them less biologically unwieldy and easier to implement [7]. As Graybiel [7] states - "It seems reasonable, given the circuitry of the basal ganglia and their apparent involvement in some neuropsychiatric disorders, that any chunking functions of the basal ganglia may also extend beyond motor chunks to a range of action repertoires."

In the light of this previous research, we sought to identify the structural volumetric neuroanatomical correlates of the frequency with which patients with schizophrenia performed discrete motor activities over a 20 hour period. To this end, we developed a novel methodology to analyse timelocked recordings of the amount of such movement, so that a measure of movement complexity could be extracted.

\section{MATERIALS AND METHODOLOGY}

\section{Patients}

Sixteen right-handed male patients with chronic, stable schizophrenia (DSM-IV; [15]) gave written informed consent and participated in the study, approved by the North Sheffield Research Ethics Committee. This was the same cohort of patients used in our previous study examining the relationship between amount of movement and volume of anterior cingulate cortex [2] and the effects of modafinil on unconstrained motor activity [3]. All patients were community-based outpatients but for monitoring purposes were admitted overnight on two separate occasions to a psychiatric ward. Subjects were reimbursed $£ 100$ for their participation in the entire study.

\section{Clinical Ratings and Medication}

Patients were $36 \pm 8$ years old (mean \pm SD), had an illness duration of $14 \pm 8$ years, a Scale for the Assessment of Negative Symptoms (SANS; [1]) score of 11.5 \pm 2.7 (the sum of 'total' scores), a Scale for the Assessment of Positive Symptoms (SAPS; [16]) score of 3.6 \pm 2.1 , a Beck Depression Inventory (BDI; [17]) score of 9.5 \pm 10.3 , a Barnes Akathisia Rating Scale (BARS; [18]) score of 1.2 \pm 1.7 and a SimpsonAngus Scale (SAS; [19]; a measure of neuroleptic-induced parkinsonism) score of $6.1 \pm 8.5$. Eleven patients were taking oral atypical antipsychotic medication (olanzapine x 6, clozapine $\mathrm{x} 4$, quetiapine $\mathrm{x} 1$ ), one was taking oral typical medication (sulpiride) and four were receiving 'typical' medication as depot (flupenthixol decanoate x 2, fluphenazine decanoate $\mathrm{x} 1$, zuclopenthixol decanoate $\mathrm{x} 1$ ).

\section{Magnetic Resonance Imaging}

Patients underwent a structural MRI scan on a $1.5 \mathrm{~T}$ system (Eclipse, Philips Medical Systems, Ohio) using a 3 dimensional acquisition technique (RF-spoiled FAST; $\mathrm{TR}=15 \mathrm{~ms} ; \mathrm{TE}=4.4 \mathrm{~ms} ;$ acquisition matrix $=256 \times 256 \times 190$ yielding a voxel size of $1 \mathrm{~mm}^{3}$ ), which produced a T1weighted, volume dataset covering the entire brain.

\section{Movement Data Collection}

Patients returned to the ward following the scan at approximately midday, where they were free to fill the remaining time as they chose. This would have included eating lunch and dinner and sleeping for one night. During this post-scan $20 \mathrm{hr}$ period, patients wore an 'Actiwatch' (Cambridge Neurotechnology Ltd, UK) measuring their cumulative activity. The Actiwatch is a wrist-worn device containing a miniature uniaxial accelerometer which produces a digital integration of the amount and duration of all movement over $0.05 \mathrm{~g}$. Individual (dimensionless) actiwatch data $(106,722 \pm 36,553$ [mean \pm SD]; range 39,614 to 171,790) were normalised to percentage measures to facilitate direct inter-subject comparisons of the distribution and variability of movement over time (Fig. 1). Subsequently, these normalised time-series data were analysed to compute the number of 'on' and 'off' movement epochs as a measure of the extent of spontaneous activity and, hence, by implication, goal-directed behaviour. Specifically, for each subject, total number of discrete blocks of activity was calculated by summation of non-contiguous points in the array representing the temporal position of non-zero elements in the raw data.

\section{Image Analysis}

Structural MRI scans were pre-processed using voxelbased morphometry [20] and the optimised protocol [21], including modulation with Jacobian determinants for the effects of spatial normalisation, in SPM2 (Wellcome Department of Imaging Neuroscience, London). A studyspecific local group template, created from the averaged structural-MRI scans of 59 patients with schizophrenia collected on the same scanner was smoothed with a Gaussian kernel of $8 \mathrm{~mm}$ full-width at half maximum. Smoothed greymatter segmented maps were entered into a simple regression model within SPM2 correlating regionally specific greymatter volumes with number of discrete movement epochs. Areas of significant correlation ( $\mathrm{p}=0.005$ uncorrected; extent 

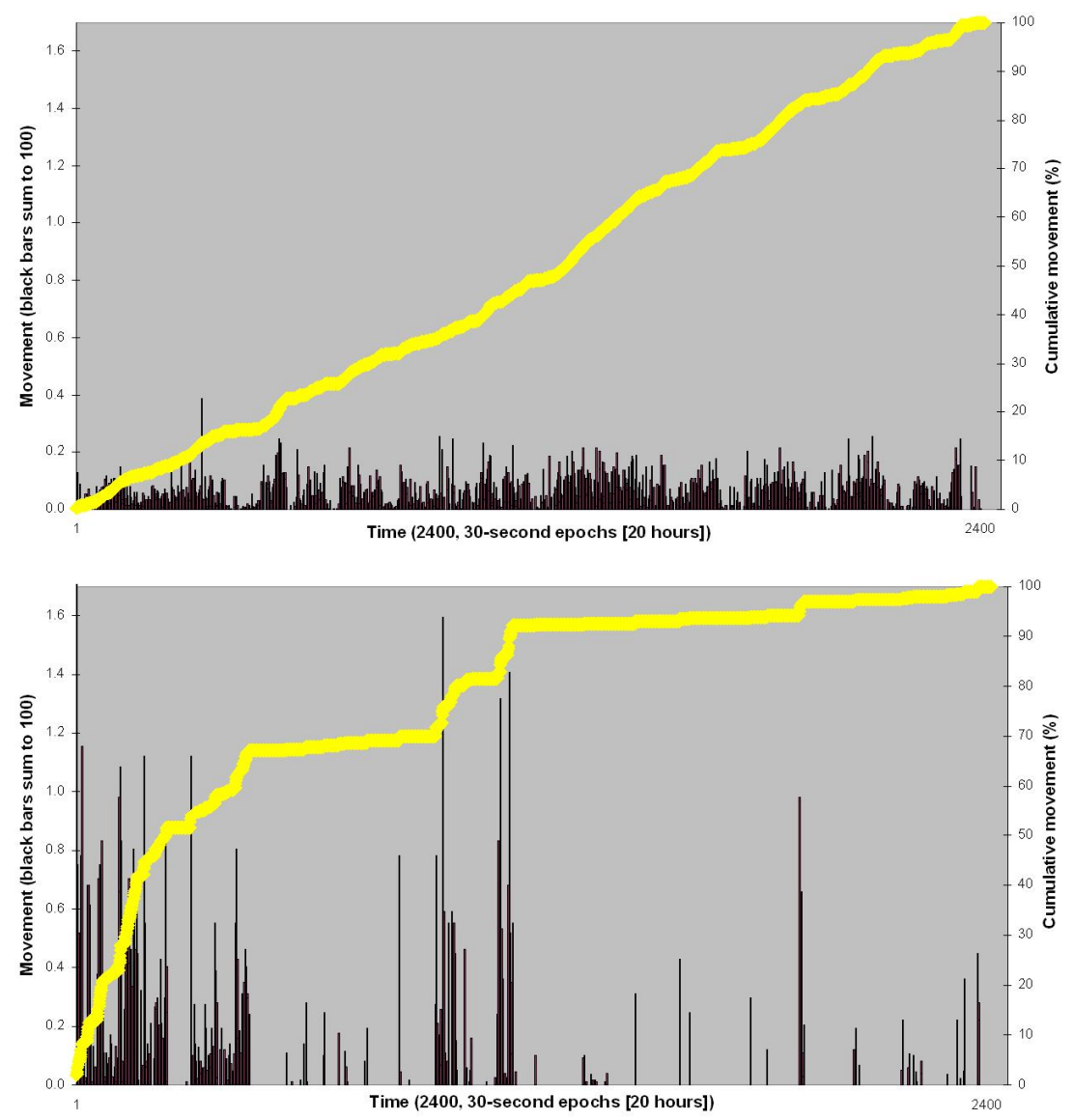

Fig. (1). Examples of 'low' variability (top) and 'high' variability (bottom) actiwatch traces. Data have been normalised to $100 \%$ to facilitate direct between-subject comparison. Thus vertical black bars (left hand Y-axis) sum to 100, and yellow line (right hand Y-axis) ascends to $100 \%$ for each subject across 2400 , thirty-second epochs (i.e. 20 hours).

threshold $=50$ voxels) were overlaid on the study-specific local group template (Fig. 2) for accurate neuroanatomical localisation and confirmed via a standardised co-planar stereotaxic brain atlas (Table 1; [22]). Grey-matter segmented maps were parcellated using masks created with WFU_PickAtlas v1.02 [23]. Volumes of left and right caudate and putamen grey-matter were obtained for each subject. Finally, these parcellated basal-ganglia volumes were compared with those of 115 healthy control subjects collected on the same scanner with the identical sequence acquisition.

\section{RESULTS}

Patients exhibited $245 \pm 62$ (mean $\pm \mathrm{SD}$; range 111 to 355 ) discrete movement epochs over 20 hours. There was a positive correlation between number of discrete movement epochs and volumes of specific homologous regions within bilateral rostro-ventral putamen and bilateral middle and superior temporal gyri (Table $\mathbf{1}$ and Fig. 2). There were no negative correlations between any regional brain volumes and number of discrete movement epochs. There were no significant correlations between number of discrete movement epochs and total volume of movement, age, duration of illness, chlorpromazine equivalent anti-psychotic medication dosage, SANS 'avolition', BARS (akathisia) score, SAS (neuroleptic-induced parkinsonism) or parcellated volumes of whole caudate or putamen. Patients' parcellated basal ganglia grey-matter volumes did not differ significantly from those of 115 healthy controls in respect of left putamen $(4.23 \pm 0.42 \mathrm{mls}$ vs. $4.18 \pm 0.42 \mathrm{mls}[$ mean $\pm \mathrm{SD}] ; \mathrm{p}=0.65)$, right putamen $(4.55 \pm 0.51$ vs. $4.64 \pm 0.41 ; \mathrm{p}=0.51)$ or right caudate $(3.85 \pm 0.43$ vs. $3.64 \pm 0.59 ; \mathrm{p}=0.09)$, although patients did have a significantly smaller volume of left caudate $(3.82 \pm 0.42$ vs. $3.53 \pm 0.47 ; p=0.02)$. However, this final result should be interpreted within the context of all 16 patients' left caudate volumes lying within the range of the 115 healthy control subjects' left caudate volumes.

\section{DISCUSSION}

In these people with schizophrenia, complexity of spontaneous motor activity (i.e. number of discrete movement epochs) over a $20 \mathrm{hr}$ period was correlated with the volume of specific homologous regions within bilateral rostro-ventral putamen, a finding supportive of our central hypothesis that anatomy may constrain function, in this case the frequency of spontaneous motor behaviour. Specifically, our findings would support evidence that sub-cortical 'bottom-up' structures in the fronto-striatal loops may modulate complex behaviours often attributed to more executive areas such as dorso-lateral prefrontal cortex (novelty generation [24]) and orbitofrontal cortex (reward seeking behaviour $[12,25])$. Our behavioural data also show that there is considerable variation across subjects over a day. Some exhibit constant increases with no discernable 

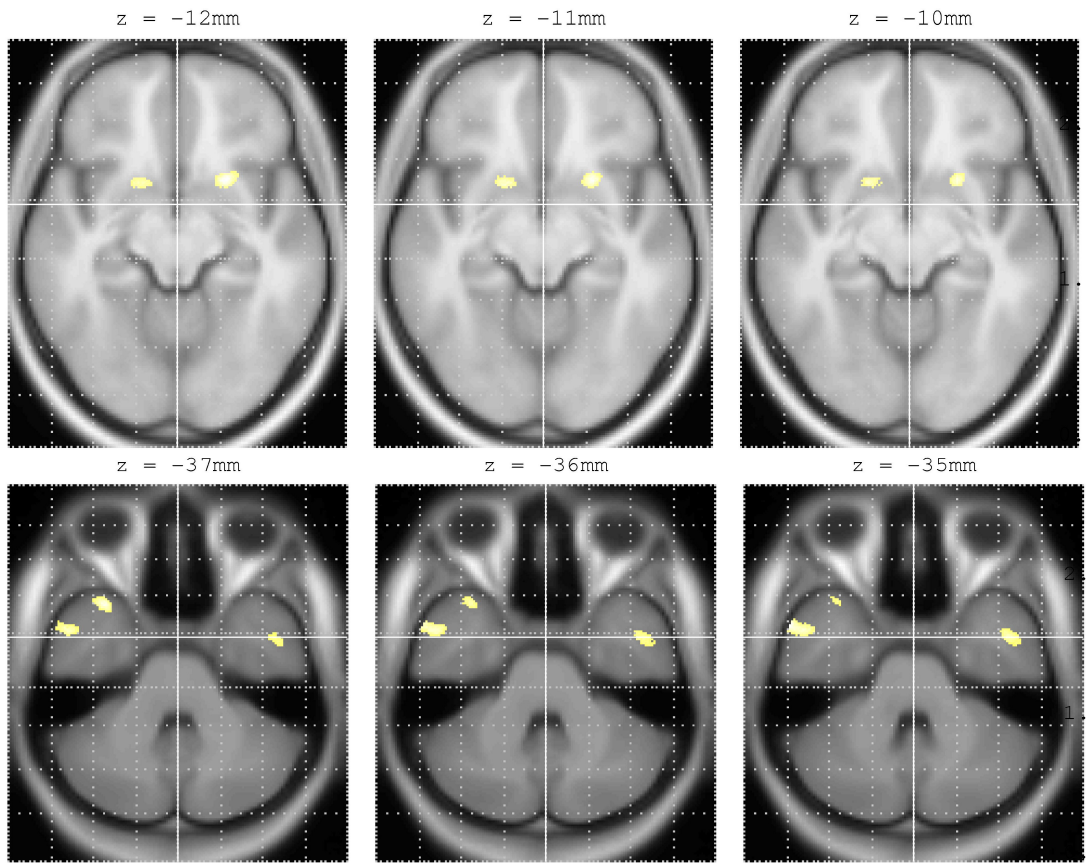

Fig. (2). Bilateral rostro-ventral putamen (top row) and middle / superior temporal gyrus (bottom row) volumes positively correlated with number of discrete movement epochs in 16 male patients with schizophrenia. Areas of correlation are overlaid on a study-specific template for accurate anatomical localisation. Co-ordinates in standardised neuroanatomical space [22] are shown in Table 1.

'chunking', whereas others exhibit discrete periods of activity (Fig. 1).

Actigraphy has previously been used to examine diurnal variations in locomotor activity [26] and neuroleptic induced akathisia [27] in patients with schizophrenia, and akathisialike hyperactivity in antisocial violent offenders with ADHD [28]. Its use as a general tool in human psychopharmacology studies has also been advocated [29]. However, in terms of methodology, ours is the first study to analyse actiwatch data in this way, to obtain the structure of activity from a time series of 'amount of movement'. This type of measure may be of utility in situations in which behavioural change is anticipated (e.g. with behavioural or pharmacological interventions), as well as a way of further exploring human voluntary behaviour in health and disease. We acknowledge that to truly assess the extent of goal-directness of a person's behaviour over 20 hours would be enormously resourceintense, requiring specialist equipment (e.g. CCTV monitoring) and complex, probably hand scored qualitative analysis. Our intention in the present study was to report a simple, cheap, quantitatively analysable methodology, which may reflect certain aspects of goal-directed behaviour.

Though phenomenological descriptions of avolitional behaviour and modular models of basal ganglia function may not correspond exactly with the underlying neurophysiology, our results are interpretable in the context of such descriptions and models. Our rostro-ventral putamen region would be implicated in 'reinforcement learning' [10], particularly if the 'goal' in 'goal-directed behaviour' is conceptualised as a reward [11]. Equally in the 'actor-critic' model of basal-ganglia function [10], the implication of our putamen finding would be an abnormality of the 'critic' function, with avolition comprising a problem maintaining learned information to modulate future choices. Our findings are also in line with the 'complexity' versus 'movement frequency' distinction between posterior and anterior putamen suggested by Lehéricy and colleagues [14] (though note the distinction in our definition of 'complexity').

Table 1. Regionally Distinct Brain Areas which Positively Correlated with Number of Discrete Movement Epochs (See Also Fig. 2)

\begin{tabular}{|c|c|c|c|c|c|c|}
\hline Anatomical Region & BA & $\mathbf{x}$ & $\mathbf{y}$ & $\mathbf{z}$ & Z-Value & Extent \\
\hline \hline Left putamen & - & -22 & 9 & -10 & 2.88 \\
\hline Right putamen & - & 19 & 9 & -9 & 2.73 \\
\hline Left MTG & 21 & -41 & 0 & -28 & 2.79 & 394 \\
\hline Right $M T G$ & 21 & 57 & 4 & -27 & 3.30 & 3.05 \\
\hline Left $S T G$ & 38 & -34 & 8 & -36 & 3.33 \\
\hline Right $S T G$ & 38 & 33 & 14 & -35 & 207 \\
\hline
\end{tabular}

Co-ordinates are shown in standardized neuroanatomical space [22]. BA = Brodmann's area, MTG = middle temporal gyrus. STG $=$ superior temporal gyrus . 
The issue of phenomenological descriptions of avolitional behaviour is also reflected in the language used. Throughout this paper we have intentionally used a number of terms to describe aspects of movement including 'structure', 'complexity', 'chunking', 'goal-directness' and 'spontaneous'. Rather than suggesting that these terms are interchangeable, we would view them as overlapping concepts describing behaviours originating from fronto-striatal circuitry, all of which are contained in the clinical assessment of avolition.

While the finding of a relationship between bilateral rostro-ventral putamen volume and motoric activity is consistent with our hypothesis, the finding of additional homologous bilateral temporal pole (middle and superior temporal gyri) correlations is more difficult to interpret in the light of previous literature. To be rather speculative, this correlation might implicate limbic inputs into the decision making process (i.e. affective saliency / valency of context) influencing movement, or may reflect an interaction with the basal ganglia themselves, specific to biological movements and the 'mirror neuron' system [30]. However, it should also be borne in mind that this data set derives from patients with schizophrenia, so the finding may be a reflection of the abnormal correlations between disparate brain region volumes known to exist, or the disturbed structure-function relationships previously described, in this disorder [31].

The lack of correlation between number of discrete movement epochs and total volume of movement, age, duration of illness, SANS 'avolition' score, BARS (akathisia) score, SAS (neuroleptic-induced parkinsonism) or medication dosage suggests that our extracted 'structure of movement' variable is independent of all these potentially confounding variables. The lack of correlation between SAS scores and volume of movement in schizophrenia has been reported before [32] using lower-limb actometer recordings during "controlled rest activity". This lack of correlation also suggests, counterfactually, that those who merely increase their activity over a day, at a constant rate, with little discernible structure, are not exhibiting akathisia. However, as a negative finding, this result requires replication. That the parcellated volumes of whole basal ganglia nuclei did not correlate with number of discrete movement epochs is probably a consequence of them including a much larger area than that identified in the SPM regression analysis. Our preliminary investigation has been exploratory, relying on uncorrected whole-brain VBM analyses and deliberately data led. In the circumstances, region of interest analysis of such a small area of rostro-ventral putamen would be extremely difficult and also particularly susceptible to scan-normalisation errors which are apparent in sub-cortical structures [33]. Our data implicate very specific regions of the putamen in the modulation of daytime activity structure.

We must be cautious however in extrapolating our findings to the community, ambulatory patient as our study concerned subjects who spent the days in question on a psychiatric ward. Furthermore, our sample size is relatively small and may therefore limit the reliability of the regression analyses performed. Nevertheless, our data do offer the intriguing possibility that anatomy and function are related with respect to spontaneous, unconstrained motor activity.
Finally, the issue of medication effects upon the basal ganglia volumes is important to consider. One previous study [34] reported significantly increased volumes of caudate $(9.5 \%)$ and putamen $(15.9 \%)$ in patients with schizophrenia and further reported that these increases were associated with poorer neuropsychological test performance. Another study [35], reported more modest increases in caudate and putamen volumes in chronically treated patients, but no difference in either volume when first-episode patients were scanned at baseline (compared with healthy controls) or any change in volumes following one-year of continuous treatment with an atypical antipsychotic. Our mean parcellated volumes of putamen and caudate were not significantly different in the main from 115 healthy controls' volumes, and where they were (in left caudate), schizophrenic individuals' data points still lay within the 'normal' range. Hence, while our findings specifically concern people with schizophrenia, they do not appear solely connected to their treatment.

In summary, our findings suggest that the volumes of specific homologous regions within bilateral rostro-ventral putamen in people with chronic schizophrenia correlate with an objective measure of the complexity of their unconstrained motor activity, independent of the volume of that activity. Our data add to the growing body of evidence that disorders of the basal ganglia may contribute to frontostriatal neural circuit dysfunctions expressed as positive and negative symptoms of schizophrenia. In our sample, those with smaller rostro-ventral putamen exhibit less structure in their spontaneous behaviour.

\section{ACKNOWLEDGEMENTS}

This study was funded by an investigator-led grant awarded to SAS by Cephalon UK. We thank nursing staff from Rowan Ward, The Longley Centre, Northern General Hospital, Sheffield. UK, and also Dr. Russell Green for his assistance with data acquisition.
ABBREVIATIONS
$\mathrm{BA}=$ Brodmann's area
BARS $=$ Barnes akathisia rating scale [18]
BDI $=$ Beck depression inventory [17]
DSM = Diagnostic and statistical manual (of mental disorders) [15]
$\mathrm{fMRI}=$ Functional magnetic resonance imaging
MTG = Middle temporal gyrus
PET = Positron emission tomography
RF-FAST $=$ Radiofrequency-spoiled - fast acquisition at steady rate
$\mathrm{ADHD}=$ Attention deficit hyperactivity disorder
SANS $=$ Scale for the assessment of negative symptoms [1]
SAPS $=$ Scale for the assessment of positive symptoms [16]
SAS $\quad=$ Simpson Angus scale [19] 


$$
\begin{array}{ll}
\mathrm{SPM} & =\text { Statistical parametric mapping } \\
\mathrm{STG} & =\text { Superior temporal gyrus } \\
\mathrm{TE} & =\text { Echo time } \\
\mathrm{TR} & =\text { Repetition time }
\end{array}
$$

\section{REFERENCES}

[1] Andreasen NC. The Scale for the Assessment of Negative Symptoms (SANS). Iowa City, IA: The University of Iowa 1983.

[2] Farrow TFD, Hunter MD, Wilkinson ID, Green RDJ, Spence SA. Structural brain correlates of unconstrained motor activity in people with schizophrenia. Br J Psychiatry 2005; 187: 481-2.

[3] Farrow TFD, Hunter MD, Haque R, Spence SA. A double-blind placebo-controlled cross over study of modafinil's effect on unconstrained motor activity in people with schizophrenia. Br J Psychiatry 2006; 189: 461-2.

[4] Barnes TRE, Spence SA. Movement disorders associated with antipsychotic drugs: clinical and biological implications. In: Waddington JL, Deakin JFW, Eds. Psychopharmacology of schizophrenia. London: Arnold 2000; pp. 178-210.

[5] Haruno M, Kawato M. Different neural correlates of reward expectation and reward expectation error in the putamen and caudate nucleus during stimulus-action-reward association learning. J Neurophysiol 2006; 95: 948-59.

[6] Graybiel AM. The basal ganglia and cognitive pattern generators. Schizophr Bull 1997; 23: 459-69.

[7] Graybiel AM. The basal ganglia and chunking of action repertoires. Neurobiol Learn Mem 1998; 70: 119-36.

[8] Jueptner M, Stephan KM, Frith CD, Brooks DJ, Frackowiak RSJ, Passingham RE. Anatomy of motor learning. I. Frontal cortex and attention to action. J Neurophysiol 1997; 77: 1313-24.

[9] Jueptner M, Frith CD, Brooks DJ, Frackowiak RSJ, Passingham RE. Anatomy of motor learning. II. Subcortical structures and learning by trial and error. J Neurophysiol 1997; 77: 1325-37.

[10] O’Doherty J, Dayan P, Schultz J, Deichmann R, Friston K, Dolan RJ. Dissociable roles of ventral and dorsal striatum in instrumental conditioning. Science 2004; 304: 452-4

[11] Yamada H, Naoyuki M, Kimura M. Tonically active neurons in the primate caudate nucleus and putamen differentially encode instructed motivational outcomes of action. J Neurosci 2004; 24: 3500-10.

[12] Robbins TW, Everitt BJ. Neurobehavioural mechanisms of reward and motivation. Curr Opin Neurobiol 1996; 6: 228-36.

[13] Canales JJ, Graybiel AM. A measure of striatal function predicts motor stereotypy. Nat Neurosci 2000; 3: 377-83.

[14] Lehéricy S, Bardinet E, Tremblay L, et al. Motor control in basal ganglia circuits using fMRI and brain atlas approaches. Cereb Cortex 2006; 16: 49-61.

[15] American Psychiatric Association. Diagnostic and statistical manual of mental disorders (DSM-IV) $4^{\text {th }}$ ed. Washington, DC: APA 1994.
[16] Andreasen NC. The Scale for the Assessment of Positive Symptoms (SAPS). Iowa City, IA: The University of Iowa 1994.

[17] Beck AT, Steer RAQ, Brown GK. Manual for Beck Depression Inventory-II. San Antonio (TX): Psychological Corporation 1996

[18] Barnes TRE. A rating scale for drug-induced akathisia. $\mathrm{Br} J$ Psychiatry 1989; 154: 672-6.

[19] Simpson GM, Angus JWS. A rating scale for extrapyramidal side effects. Acta Psychiatr Scand 1970; 212: 11-9.

[20] Ashburner J, Friston KJ. Voxel-based morphometry - the methods. NeuroImage 2000; 11: 805-21.

[21] Good CD, Johnsrude I, Ashburner J, Henson RNA, Friston KJ, Frackowiak RSJ. A voxel-based morphometric study of ageing in 465 normal adult human brains. NeuroImage 2001; 14: 21-36.

[22] Talairach J, Tournoux P. Co-planar stereotactic atlas of the human brain. Stuttgart: Thieme-Verlag 1988.

[23] Maldjian JA, Laurienti PJ, Kraft R, Burdette JH. An automated method for neuroanatomic and cytoarchitectonic atlas-based interrogation of fMRI data sets. NeuroImage 2003; 19: 1233-39.

[24] Hunter MD, Green RDJ, Wilkinson ID, Spence SA. Spatial and temporal dissociation in prefrontal cortex during action execution. NeuroImage 2004; 23: 1186-91.

[25] Rolls ET. The orbitofrontal cortex and reward. Cerebral Cortex 2000; 10: 284-94.

[26] Poyurovsky M, Nave R, Epstein R, et al. Actigraphic monitoring (actigraphy) of circadian locomotor activity in schizophrenic patients with acute neuroleptic-induced akathisia. Eur Neuropsychopharmacol 2000; 10: 171-6.

[27] Tuisku K, Lauerma H, Holi M, Markkula J, Rimon R. Measuring neuroleptic-induced akathisia by three-channel actometry. Schizophr Res 1999; 40: 105-10.

[28] Tuisku K, Virkkunen M, Holi M, et al. Antisocial violent offenders with attention deficit hyperactivity disorder demonstrate akathisialike hyperactivity in three-channel actometry. J Neuropsychiatry Clin Neurosci 2003; 15: 194-9.

[29] Stanley N. Actigraphy in human psychopharmacology: a review. Hum Psychopharmacol: Clin Exp 2003; 18: 39-49.

[30] Kessler K, Biermann-Ruben K, Jonas M, et al. Investigating the human mirror neuron system by means of cortical synchronization during the imitation of biological movements. NeuroImage 2006; 33: 227-38

[31] Burns J, Job D, Bastin ME, et al. Structural disconnectivity in schizophrenia: a diffusion tensor magnetic resonance imaging study. Br J Psychiatry 2003; 182: 439-43.

[32] Janno S, Holi MM, Tuisku K, Wahlbeck K. Validity of SimpsonAngus scale (SAS) in a naturalistic schizophrenia population. BMC Neurol 2005; 5: 5.

[33] Shen S, Szameitat A, Sterr A. VBM lesion detection depends on the normalization template: a study using simulated atrophy. Magn Reson Imaging 2007; 25: 1385-96

[34] Hokama H, Shenton ME, Nestor PG, et al. Caudate, putamen, and globus pallidus volume in schizophrenia: a quantitative MRI study. Psychiatry Res 1995; 61: 209-29.

[35] Lang DJ, Kopala LC, Vandorpe RA, et al. An MRI study of basal ganglia volumes in first-episode schizophrenia patients treated with risperidone. Am J Psychiatry 2001; 158: 625-31.

(C) Farrow et al.; Licensee Bentham Open.

This is an open access article licensed under the terms of the Creative Commons Attribution Non-Commercial License (http://creativecommons.org/licenses/by-nc/3.0/) which permits unrestricted, non-commercial use, distribution and reproduction in any medium, provided the work is properly cited. 